\title{
Water/oil microemulsion for the preparation of robust La-hexaaluminates for methane catalytic combustion
}

\author{
Zheng Jiang, ${ }^{a}$ Zhengping Hao, ${ }^{b}$ Jixi Su, ${ }^{c}$ Tiancun Xiao ${ }^{* a}$ and Peter P. Edwards ${ }^{* a}$ \\ Received (in Cambridge, UK) 5th January 2009, Accepted 6th March 2009 \\ First published as an Advance Article on the web 20th April 2009 \\ DOI: $10.1039 / b 900012 \mathrm{~g}$
}

Robust and highly effective Fe-substituted and non-substituted La-Hexaaluminate methane combustion catalysts have been prepared via coprecipitation in "water-pools" of Water/Oil microemulsions with dual nonionic surfactants, followed by ethanol supercritical drying and post-annealing.

Compared with conventional (homogenous) combustion, catalytic combustion of methane in a gas turbine provides a significantly more economic and efficient solution for power generation with ultralow emissions of environmental pollutants, such as NOx, CO, and UHCs (unburned hydrocarbons). ${ }^{1}$ Although noble metal and perovskite materials are all active combustion catalysts, they do not match the stringent requirements of this combustion process owing to their serious deactivation at high temperature. ${ }^{1-4}$ To date, hexaaluminate $\left(\mathrm{HA}, \mathrm{BAl}_{12 \pm x} \mathrm{O}_{19 \pm \delta}, x=0 \sim 2, \mathrm{~B}=\right.$ alkaline or rare earth metal having a large ionic radius) has been regarded as the best catalytic material for tolerating the severe hydrothermal conditions of catalytic combustion. ${ }^{3}$ However, the ignition activity of common HA's is far from ideal.

Extensive efforts have been made to improve the catalytic reactivity of HA's by incorporating transition metals (TM's) into HA matrices or adopting advanced synthesis methods (sol-gel, supercritical drying and reverse microemulsion synthesis, etc). $\mathrm{Mn}$ and $\mathrm{Fe}$ are the best candidates among the TM's to promote the catalytic combustion activity of HA through a partial substitution route. ${ }^{3,5}$ Unfortunately, TM-substitution usually leads to a significant decrease in the specific surface area (SSA) of HA and a transportation limitation for gaseous reactants., ${ }^{3,6}$ A breakthrough has been achieved by Zarur and Ying ${ }^{2,7,8}$ on the synthesis of barium hexaaluminate (BHA) via reverse microemulsion synthesis (RM) followed by supercitical drying (SCD) and postannealing. Their RM-SCD $\mathrm{CeO}_{2} / \mathrm{BHA}$ catalysts possessed extremely high activity and excellent thermal stability. ${ }^{2}$ Sahu and coworkers ${ }^{9}$ prepared BHA nanowhiskers with high SSA via a modified sol-gel-RM route using Triton X-100 as surfactant (hereafter, saa). So far, the RM synthesis strategy has been extensively conducted to prepare HA's or TM-substituted HA's. ${ }^{2,-14}$ However, most of the RM synthesis routes

${ }^{a}$ Department of Chemistry, Inorganic Chemistry Laboratory,

University of Oxford, Oxford, UK OX1 3QR.

E-mail:peter.edwards@chem.ox.ac.uk,xiao.tiancun@chem.ox.ac.uk,

Fax: + 441865 272656; Tel: + 441865272646

${ }^{b}$ Research Centre for Eco-Environmental Sciences, CAS, Beijing,

100085, China.E-mail: zpinghao@rcees.ac.cn;

Tel: +861062849194

${ }^{c}$ School of Environmental Science and Engineering,

Shandong University, Jinan, 250100 Shandong, China

involved alkoxide reactants which are expensive and moisture sensitive. For cost and environmental concerns, Teng and coworkers $^{15}$ prepared $\mathrm{Ce}_{x} \mathrm{Ba}_{1-x} \mathrm{MnAl}_{11} \mathrm{O}_{y}$ HA's using relatively inexpensive inorganic reagents in $\mathrm{RM}$ systems and found that SCD is superior to conventional drying. Their materials have high SSA and good activity for methane catalytic combustion, even though some of the $\mathrm{CeO}_{2}$ phase is isolated on HA, presumably due to a mismatch of the $\mathrm{Ce}^{\mathrm{IV}}$ radius with the host HA crystal structure. The progress on the preparation of HA's using RM synthesis suggested that the morphology and size of HA's mainly relate to the saa employed as well as the phase components (water/saa/ co-saa/oil ratio) in the RM systems. The post treatments (drying and annealing), especially SCD, play critical roles in retaining high SSA's and the final morphologies of HA's derived from the RM synthesis. ${ }^{15}$ Clearly, the innovative combination of RM synthesis with different surfactants and SCD may provide great potential for the preparation of active HA catalysts.

We report here a novel synthetic methodology called RM-CP-SCD, as shown in Fig. 1, involving dual-saa reverse microemulsion and SCD drying. The La-based hexaaluminate (LHA-RM-CP-SCD) and Fe-sub LHA (FLHA-RM-CP-SCD) have been prepared by this novel RM-CP-SCD method. The inorganic reactants are coprecipitated in the water-pools of the dual-saa RM systems, then the precipitates are centrifuged, washed, ethanol-SCD dried, and finally calcined. In the present study, two nonionic surfactants, AEO5 (R-(R- $\left(\mathrm{OCH}_{2} \mathrm{CH}_{2}\right)_{5} \mathrm{OH}$, Polyoxyethylene Fatty Alcohol Ether) and Span 40 (sorbitan monopalmitate), were used simultaneously. They have different HLB (Hydrophile-Lipophile

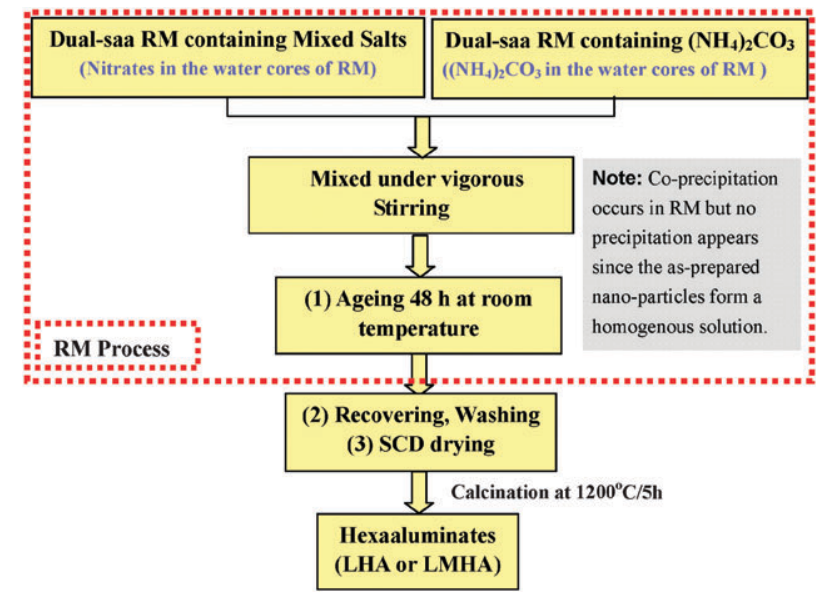

Fig. 1 General procedure for the RM-CP-SCD synthesis of $\mathrm{La}(\mathrm{M}) \mathrm{Al}-\mathrm{Hexaaluminates}$ 
Balance) values which reflect the different emulsion capabilities and determine the "water-pool" shape. Our co-surfactant is $n$-pentol and the inorganic precipitate is $\left(\mathrm{NH}_{4}\right)_{2} \mathrm{CO}_{3}$. The methods are much cheaper than the classic RM preparation and can be easily extended for the preparation of other hexaaluminates of importance.

Typically, a reverse microemulsion containing $\left(\mathrm{NH}_{4}\right)_{2} \mathrm{CO}_{3}$ $(R M \mathrm{~A})$ was added dropwise into $R M \mathrm{~B}$ containing stoichiometrical $\mathrm{La}\left(\mathrm{NO}_{3}\right)_{3}$ and $\mathrm{Al}\left(\mathrm{NO}_{3}\right)_{3}$ under vigorous stirring. The received clear RM aged for $48 \mathrm{~h}$ before centrifugation and SCD at $260{ }^{\circ} \mathrm{C} / 2 \mathrm{~h}$. The recovered aerosols were then calcined at $1200{ }^{\circ} \mathrm{C}$ for $5 \mathrm{~h}$ with a temperature ramp of $2{ }^{\circ} \mathrm{C} / \mathrm{min}$ to obtain LHA-RM-CP-SCD. For the synthesis of FLHA-RM-SCD, $R M$ B contains stoichiometric $\mathrm{La}\left(\mathrm{NO}_{3}\right)_{3}, \mathrm{Al}\left(\mathrm{NO}_{3}\right)_{3}$ and $\mathrm{Fe}\left(\mathrm{NO}_{3}\right)_{3}$ concentrations. The composition of the $\mathrm{RM}$ is saa $\%$ around $20 \mathrm{wt} \%(\mathrm{AEO} / \mathrm{Span} 40=1 / 2 \mathrm{wt}), n$-pentol about $10 \%$, water about $5-15 \%$, and the oil phase (cyclohexane) around $50-60 \%$ depending on the synthesis systems. The HA's prepared using $\mathrm{CP}$ and $\mathrm{CP}-\mathrm{SCD}$ methods were also prepared for comparison. The obtained HA's were characterized using XRD, TEM and $\mathrm{N}_{2}$ adsorption-desorption. The lean methane catalytic combustion tests were conducted in a fixed bed micro-reactor as described previously: ${ }^{16} 200 \mathrm{mg}$ catalyst mixed with $200 \mathrm{mg}$ silica (40-60 mesh) were mounted in a quartz tube (4 mm inner diameter), with the $\mathrm{CH}_{4} / \mathrm{air}=1: 99$ (vol), the gas mixture flow rate was $6 \mathrm{~L} \mathrm{~h}^{-1} \mathrm{~g}^{-1}$ catalyst. The exit gases were detected using online gas chromotagraphy.

In Fig. 2, we show the powder XRD patterns of the LHA- and FLHA-RM-CP-SCD samples. The only phases observed are pure and Fe-substituted La-hexaaluminate phases (JCPDF:84-1978) and there are no detectable impurity phases. This is evidence that $\mathrm{Fe}$ has been fully incorporated into the hexaaluminate matrix. The stronger XRD reflection of the FLHA-RM-CP-SCD suggests that the Fe-substitution remarkably improves the crystallity of the hexaaluminate.

To study the effects of the preparation method on catalyst activity, we have compared the methane catalytic combustion over Fe-Sub and pure LHA's prepared using different methods, and these are shown in Fig. 3. The characteristic methane conversion temperatures are listed in Table 1 . We notice that the activities of the samples prepared using the RM-CP-SCD method are much higher than the corresponding catalysts prepared using CP-SCD or coprecipitation (CP) routes. Moreover, we find that SCD has more advantages

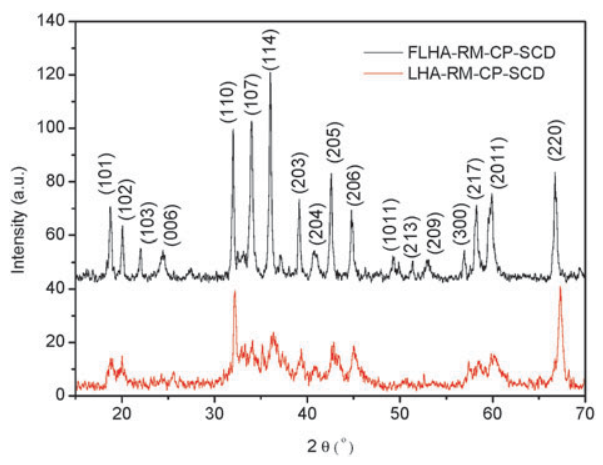

Fig. 2 XRD patterns of FLHA-RM-CP-SCD and LHA-RM-CPSCD.

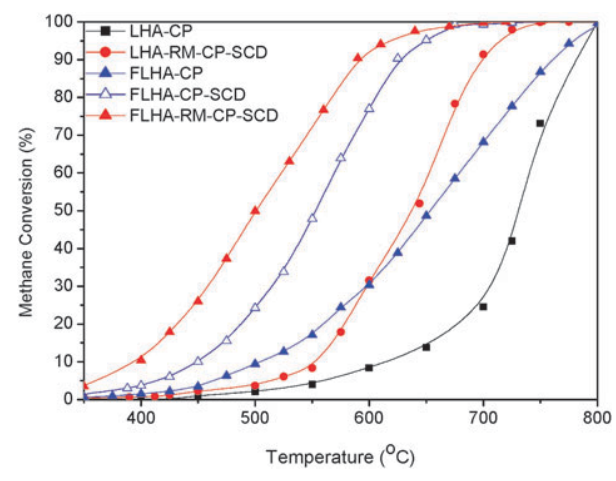

Fig. 3 Methane catalytic combustion activity over LHA and FLHA.

Table 1 SSAs and activities of La-based hexaaluminate catalysts

\begin{tabular}{lllll}
\hline Samples & $\begin{array}{c}\mathrm{SSA} \\
\left(\mathrm{m}^{2} / \mathrm{g}\right)\end{array}$ & $\begin{array}{l}T_{10} \\
\left({ }^{\circ} \mathrm{C}\right)\end{array}$ & $\begin{array}{l}T_{50} \\
\left({ }^{\circ} \mathrm{C}\right)\end{array}$ & $\begin{array}{l}T_{90} \\
\left({ }^{\circ} \mathrm{C}\right)\end{array}$ \\
\hline LHA-CP & 23.7 & 620 & 725 & 775 \\
LHA-RM-CP-SCD & 55.3 & 550 & 635 & 690 \\
FLHA-RM-CP-SCD & 45.5 & 400 & 495 & 590 \\
FLHA-CP-SCD & 21 & 445 & 550 & 625 \\
FLHA-CP & 11.5 & 505 & 654 & 761 \\
Pd $(1 \mathrm{wt} \%) / \mathrm{Al}_{2} \mathrm{O}_{3}$ & 138 & 420 & 475 & 550 \\
Silica & - & 765 & - & 790 \\
\hline
\end{tabular}

over conventional drying on the reactivities of La-based HA's. The FLHA-RM-CP-SCD material shows the best catalytic activity, on which methane ignites and completely combusts at about $400{ }^{\circ} \mathrm{C}$ and $590{ }^{\circ} \mathrm{C}$, respectively, which are considerably lower temperatures than those on FLHA-CP-SCD and FLHA-CP. Importantly, its activity is comparable to noble metal catalysts, but markedly more stable considering the higher calcination temperature. In particular, no deactivation has been observed in a $100 \mathrm{~h}$ stability test at $600{ }^{\circ} \mathrm{C}$ (continuous combustion over FLHA-RM-CP-SCD).

Since methane catalytic combustion at a higher conversion and temperature is diffusion controlled, the reaction rate depends not only on the number of catalytically active sites, but also on the available surface area. Therefore, thermally stable catalysts with high SSA's are essential. The SSA's of as-prepared catalysts are also listed in Table 1. The data clearly show that RM-CP-SCD is efficient for the preparation of hexaaluminates with high SSAs. SCD can also greatly improve the SSA of the resulting materials. The activity and SSA results indicate that the RM synthesis and SCD are initial factors determining the performances and properties of these LHA and FLHA catalysts. This is probably as a result of two aspects: (1) the "water-pools" of RM serve as mini reactors for subsequent coprecipitation, which inhibit the aggregation of the as-coprecipited and monodispersed precursor particles; (2) the disappearance of gas-liquid-solid phase interfaces and etherification of surface $-\mathrm{OH}$ on precursors during supercritical drying, which reduces their agglomeration and thus inhibits catalyst sintering in the subsequent calcination. The beneficial effect of SCD is clear by comparing the SSA's of samples prepared using CP-SCD and CP methods (Table 1).

In order to understand the synthesis mechanism of RM-CPSCD, TEM characterizations were carried out. The morphologies of recovered particles and calcined samples $\left(500^{\circ} \mathrm{C}\right)$ are shown 


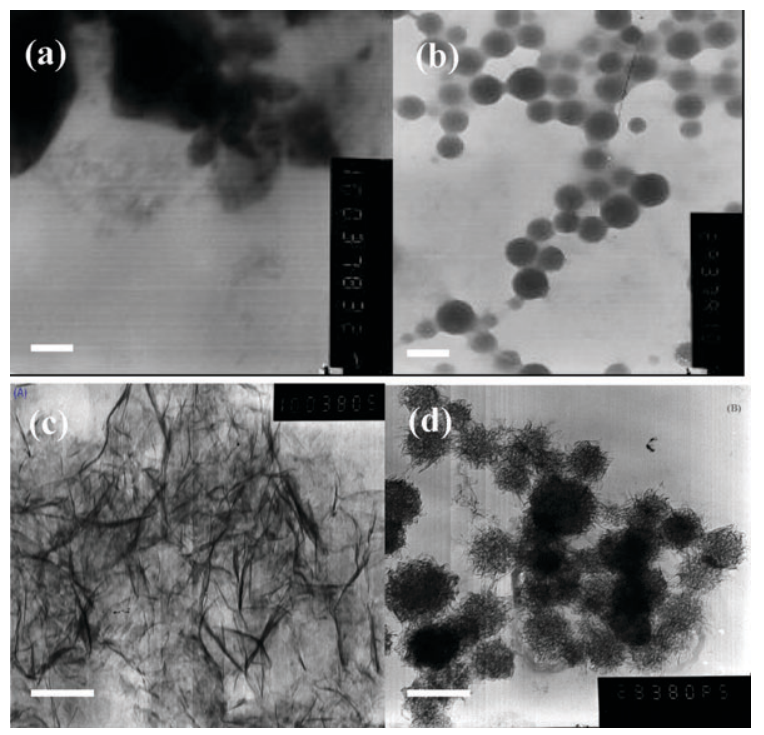

Fig. 4 TEM images of precursors of LHA (as-prepared LHA precursors prepared using (a) co-precipitated.; (b) RM methods. Calcined LHA precursors at $500^{\circ} \mathrm{C}$ : (c).LHA-CP-SCD, (d) LHA-RM-CP-SCD). Scale bars: $100 \mathrm{~nm}$.

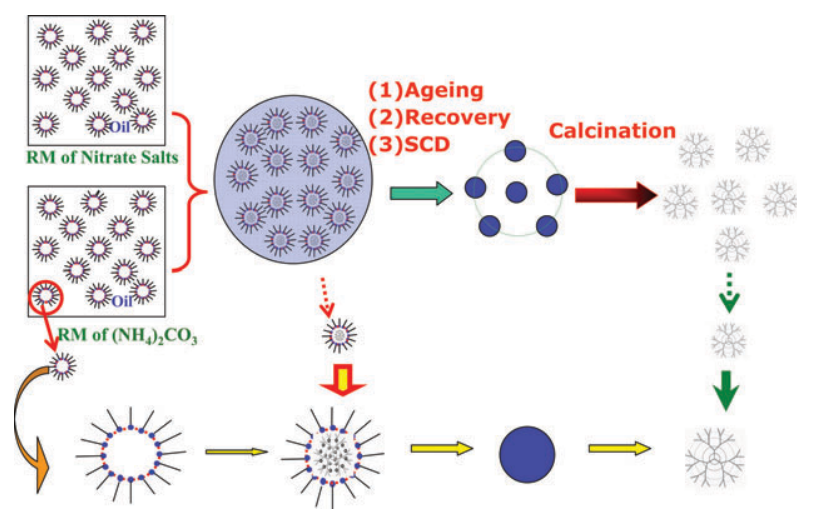

Fig. 5 Proposed synthesis mechanism of the RM-CP-SCD method.

in Fig. 4 (a,b: Precursors; c,d: calcined samples), respectively. Discrete nano-spheres with diameters about $70 \mathrm{~nm}$ are observed for the precursor as-recovered from the RM system. However, strong aggregation with bulk particles is revealed for the CP-derived precursor. The significant influence of SCD treatment is also observed for the samples after SCD treatment and calcination at $500{ }^{\circ} \mathrm{C}$. The LHA-SCD sample has the morphology of nanowhiskers whereas the LHA-RM-CP-SCD one maintains dispersed spheres constructed with finite fibers. The high aspect ratio of the fibers is helpful in depressing sintering under high temperature calcinations, and thus enhancing their combustion activity.

In Fig. 5, we illustrate a tentative synthesis mechanism of nano-structured LHA's-RM-CP-SCD; the dual-surfactant RM systems play crucial roles for the formation of the special morphologies of precursors. The $\left(\mathrm{CH}_{2}\right) x$ chains of more lipophilic surfactant (Span 40, HLB $=6.7$ ) dispersed in the oil phase confine the water in a spherical core, and its PEO groups insert into the water core as a template for coprecipitation. The spherical water cores serve as a microreactor for coprecipitation. Therein, the mixed nitrates are coprecipitated by reaction with $\left(\mathrm{NH}_{4}\right)_{2} \mathrm{CO}_{3}$ and spherical precipitators are produced; simultaneously, PEO head groups of the more hydrophilic second surfactant (AEO-5, HLB = 10.2) also insert into the water core and serve as a second template for precursor precipitation. The enhanced stability of doublesurfactant microemulsion leads to the well-confined nano-fibers. Importantly, the SCD drying treatment extracts water and partial surfactant but does not destroy the spherical external aspect. Hence the discrete shapes of the tangled nano-fibers are clearly retained. The residual surfactants are further removed in the calcination process during the formation of the hexaaluminate phase.

In summary, the methods of RM-CP-SCD (in which coprecipitation takes place in a RM nano-reactor followed by SCD treatment) provide an efficient technology to prepare robust and stable nano-structured hexaaluminates for effective methane catalytic combustion. We believe this method can be extended to prepare other major classes of nano-structured catalytic materials having a large surface area, high stability and excellent reactivity.

This work was financially supported by the UKSHEC (EPRSC) and the National Basic Research Program of China (2004CB719500).

\section{Notes and references}

1 J. G. McCarty, Nature, 2000, 403, 35-36.

2 A. J. Zarur and J. Y. Ying, Nature, 2000, 403, 65-67.

3 T. V. Choudhary, S. Banerjee and V. R. Choudhary, Appl. Catal., A, 2002, 234, 1-23.

4 G. Guan, R. Zapf, G. Kolb, Y. Men, V. Hessel, H. Loewe, J. Ye and R. Zentel, Chem. Commun., 2007, 260-262.

5 M. Machida, K. Eguchi and H. Arai, J. Catal., 1989, 120, 377-386.

6 Z. Jiang, H. Hou, Z. Hao, S. Kang, J. Li and C. Hu, Acta. Phys.-Chim. Sin., 2004, 20, 1313-1319.

7 A. J. Zarur, H. H. Hwu and J. Y. Ying, Langmuir, 2000, 16, 3042-3049.

8 A. J. Zarur, N. Z. Mehenti, A. T. Heibel and J. Y. Ying, Langmuir, $2000,16,9168-9176$.

9 P. K. Sahu, B. D. Kulkarni, R. B. Khomane, S. A. Pardhy, U. D. Phalgune, P. Rajmohanan and R. Pasricha, Chem. Commun., 2003, 1876-1877.

10 S. Zhu, X. Wang, A. Wang, Y. Cong and T. Zhang, Chem. Commun., 2007, 1695-1697.

11 E. E. Svensson, M. Lualdi, M. Boutonnet and S. G. Jaeraas, Stud. Surf. Sci. Catal., 2007, 172, 465-468.

12 Z. You, K. Inazu, K.-I. Aika and T. Baba, J. Catal., 2007, 251, 321-331.

13 Z. You, K. Inazu, I. Balint and K.-i. Aika, Chem. Lett., 2005, 34, 692-693.

14 J. Xu, Z. Tian, Y. Xu, Z. Xu and L. Lin, Stud. Surf. Sci. Catal., 2004, 147, 481-486.

15 F. Teng, P. Xu, Z. Tian, G. Xiong, Y. Xu, Z. Xu and L. Lin, Green Chem., 2005, 7, 493-499.

16 Z. Jiang, Z. Hao, J. Yu, H. Hou, C. Hu and J. Su, Catal. Lett., $2005,99,157-163$. 